\title{
Unveiling Majorana Quasiparticles by a Quantum Phase Transition: Proposal of a Current Switch
}

\author{
F. A. Dessotti ${ }^{1}$, L. S. Ricco ${ }^{1}$, Y. Marques ${ }^{1}$, L. H. Guessi ${ }^{2,3}$, \\ M. Yoshida ${ }^{2}$, M. S. Figueira ${ }^{4}$, M. de Souza ${ }^{2}$, Pasquale Sodano ${ }^{5,6}$, and A. C. Seridonio ${ }^{1,2}$ \\ ${ }^{1}$ Departamento de Física e Química, Unesp - Univ Estadual Paulista, 15385-000, Ilha Solteira, SP, Brazil \\ ${ }^{2}$ IGCE, Unesp - Univ Estadual Paulista, Departamento de Física, 13506-900, Rio Claro, SP, Brazil \\ ${ }^{3}$ Instituto de Física de São Carlos, Universidade de São Paulo, C.P. 369, São Carlos, SP, 13560-970, Brazil \\ ${ }^{4}$ Instituto de Física, Universidade Federal Fluminense, 24210-340, Niterói, RJ, Brazil \\ ${ }^{5}$ International Institute of Physics, Universidade Federal do Rio Grande do Norte, 59078-400, Natal, RN, Brazil \\ ${ }^{6}$ Departamento de Física Teórica e Experimental, \\ Universidade Federal do Rio Grande do Norte, 59072-970 Natal, RN, Brazil
}

\begin{abstract}
We propose a theoretical approach based on an interferometer composed by two quantum dots asymmetrically coupled to isolated Majorana quasiparticles (MQPs), lying on the edges of two topological Kitaev chains, respectively via couplings $(t+\Delta)$ and $(\Delta-t)$. This setup enables us to probe MQPs in a quite distinct way from the zero-bias peak feature. Most importantly, the system behaves as a current switch made by two distinct paths: (i) for the upper dot connected to both chains, the device perceives both MQPs as an ordinary fermion and the current crosses solely the lower dot, since current in the upper dot is prevented due to the presence of the superconducting gap; and (ii) by suppressing slightly the hybridization of the upper dot with one chain, the current is abruptly switched to flow through this dot, once a trapped electron as a bound state in the continuum (BIC) [Phys. Rev. B 93, 165116 (2016)] appears in the lower dot. Such a current switch between upper and lower dots characterizes the Quantum Phase Transition (QPT) proposed here, being the ratio $t / \Delta$ the control parameter of the transition. This QPT is associated with a change from an ordinary fermionic excitation regime to a MQP in the interferometer, which enables not only the fundamental revealing of MQPs, but also yields a current switch assisted by them.
\end{abstract}

PACS numbers: 72.10.Fk 73.63.Kv 74.20.Mn

\section{INTRODUCTION}

A scenario of misinterpretations concerning the real existence of a Majorana quasiparticle (MQP) in condensed matter systems $1-\underline{3}$ is due to the demand of the zerobias peak (ZBP) $\stackrel{4.5}{n}$ as the clear evidence for its proof, once such a characteristic may stem from or masked by other phenomena $a^{\underline{6}-\underline{\underline{8}}}$ as we will discuss below. Thereby in this work, we propose an alternative strategy to avoid this demand by introducing a novel based MQP current switch by means of an emerging quantum phase transition (QPT). Particularly, a MQP attached to an edge of a topological Kitaev chain ${ }^{\underline{9}} \underline{-15}$, as known theoretically, has as fingerprint the fractional ZBP $G=0.5 e^{2} / h$ appearing in the conductance through a quantum dot $(\mathrm{QD})^{\underline{16}, 17}$. Such a signature is elusive, once other physical phenomena and experimental difficulties can mask this feature by leading to a ZBP disregarding the MQP picture. Thus some criticisms have been reported in the literature addressing the validity of the ZBPs found in the experiments of Refs. [4] and [5], respectively for semiconducting nanowire and magnetic adatom systems in the presence of strong spin-orbit and magnetic fields with an $s$-wave superconducting surface. In the experiments above, it is not clear whether the ZBPs are due to a genuine isolated MQP or associated with disorder introduced by ordinary fermionic states lying within the superconducting gap, crossed Andreev effect, among others $\mathbf{6}^{-\underline{8}}$. Furthermore, thermal broadening together with a coherence a
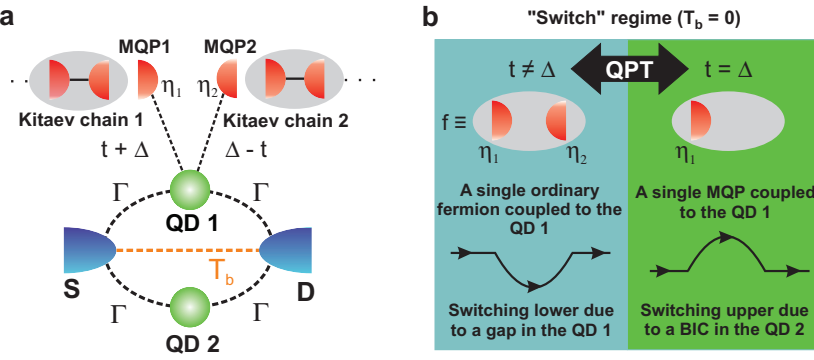

Figure 1. (Color online) (a) Double-QD interferometer connected to two Kitaev chains with MQPs $\eta_{1}$ and $\eta_{2}$. The QDs are hybridized via $\Gamma$ with the leads, while the upper QD with the chains by means of $(t+\Delta)$ and $(\Delta-t)$, respectively. $\mathcal{T}_{b}$ represents the background transmittance through the source (S) and drain (D) leads. (b) QPT: the interferometer behaves as an abrupt current switch for $\mathcal{T}_{b}=0$ when we tune from the $t \neq \Delta$ regime to $t=\Delta$.

length much longer than the Kitaev chain size can also lead to the overlap of the MQPs at the chain edges, thus suppressing the ZBP signature $\stackrel{8}{\text {. }}$.

Here our detection strategy adds a second QD to the system developed in our previous work ${ }^{18}$, where a novel technology for qubit storage was proposed based on bound states in the continuum (BICs) $\underline{19}^{\underline{21}}$ formed by MQPs. Thus we show that by using a double-QD interferometer connected to a pair of topological Kitaev chains, as those sketched in Fig प(a), that an emerging 
QPT then unveils a MQP when the setup operates under the "Switch" regime for the current, namely: if the upper QD is coupled simultaneously to the Kitaev chains, the interferometer "feels" the two MQPs at the edges of the chains just as an ordinary fermion and the current travels exclusively through the lower dot (blue panel of Fig1(b)) once the other dot presents a superconducting gap that prevents the current. By reducing slightly the coupling between one chain and the upper dot, then the current suddenly switches, the QPT here addressed, to the path via this dot (green panel of Fig[1(b)) as aftermath of the BIC rising in the lower dot that blocks the current through it, which is a straight indication of the half-fermion nature imposed by the unique MQP present in the system. Off the "Switch" regime (see Fig 4), the QPT persists still via the abrupt change in the transmittance lineshape, but revealing novel fractional values $G=0.25 e^{2} / h$ and $G=0.75 e^{2} / h$ for the MQPs.

Before starting the system analysis itself, we should call attention for the model validity within an experimental perspective, specially due to the role of disorder in realistic Kitaev chains. It is well known that $p$-wave superconductors as aftermath of its spinless superconducting nature, which is accompanied by the topological phase, is then fragile against nonmagnetic elastic scattering. There are several reports in the literature covering the disorder issue in the case of Kitaev chains. As a matter of fact, as pointed out by Refs. [6,7], there is indeed the need of having high purity chains in order to observe topological superconductivity. In quantitative terms, one has Ref. 7] pointing out that, if the ratio $\tau \gg \hbar J /\left(\Delta_{s} E_{S O}\right)$ for the elastic scattering rate is fulfilled, the topological superconductivity should remain and as expected, the QPT we have found as well, wherein $J$ represents the exchange spin splitting, $\Delta_{s}$ the $s$-wave pairing of the chain's host and $E_{S O}$ is the spin-orbit coupling energy. In general terms, the disorder is an important aspect, both on the theoretical framework ${ }^{22}$ as well as related to the fabrication of devices based on the Kitaev chains. Thus, in the present work we focus on the expected intrinsic effects of an "ideal" Kitaev chain and for this reason, we do not carried out any analysis concerning the disorder issue. More specifically, as we are dealing with a phase transition at zero temperature, quasiparticle poisoning is thus ruled out ${ }^{23}$.

\section{THEORETICAL SYSTEM}

We employ an extension of the Hamiltonian inspired on the proposal from Liu and Baranger, which is a spinless model to ensure topological superconductivity $\stackrel{16}{\underline{ }}$ :

$$
\begin{aligned}
\mathcal{H} & =\sum_{\alpha k} \tilde{\varepsilon}_{\alpha k} c_{\alpha k}^{\dagger} c_{\alpha k}+\sum_{j=1}^{2} \varepsilon_{j} d_{j}^{\dagger} d_{j}+V \sum_{\alpha k j}\left(c_{\alpha k}^{\dagger} d_{j}+\text { H.c. }\right) \\
& +V_{S D}\left(\sum_{k p} c_{S k}^{\dagger} c_{D p}+\text { H.c. }\right)+\frac{(t+\Delta)}{\sqrt{2}}\left(d_{1}-d_{1}^{\dagger}\right) \eta_{1} \\
& +i \frac{(\Delta-t)}{\sqrt{2}}\left(d_{1}+d_{1}^{\dagger}\right) \eta_{2}
\end{aligned}
$$

where the electrons in the lead $\alpha=S, D$ are described by the operator $c_{\alpha k}^{\dagger}\left(c_{\alpha k}\right)$ for the creation (annihilation) of an electron in a quantum state $k$ with energy $\tilde{\varepsilon}_{\alpha k}=\varepsilon_{k}-\mu_{\alpha}$, with $\mu_{\alpha}$ as the chemical potential. For the QDs coupled to leads, $d_{j}^{\dagger}\left(d_{j}\right)$ creates (annihilates) an electron in the state $\varepsilon_{j} . V$ (or $\Gamma=2 \pi V^{2} \rho_{0}$, the Anderson parameter ${ }^{24}$ with $\rho_{0}$ the metallic leads density of states) stands for the hybridizations between the QDs and the leads, which are considered equal to ensure the same conductance through the source and drain leads, i.e., $G=G_{S}=G_{D^{25,26}}$. The QD 1 couples asymmetrically to the Kitaev chains with tunneling amplitudes defined for convenience by $t_{L} \equiv(1 / \sqrt{2})(t+\Delta)$ and $t_{R} \equiv(i / \sqrt{2})(\Delta-t)$, respectively for the left and right MQPs $\eta_{1}=\eta_{1}^{\dagger}$ and $\eta_{2}=\eta_{2}^{\dagger}$. We stress that such definitions constitute just a choice of gauge which allows us to catch the following phenomenology: $t_{L}$ and $t_{R}$ change the last two terms of Eq. (1) into $t d_{1} f^{\dagger}+\Delta f^{\dagger} d_{1}^{\dagger}+$ H.c., when the ordinary fermion substitution $f=\frac{1}{\sqrt{2}}\left(\eta_{1}+i \eta_{2}\right)$ and $f^{\dagger}=\frac{1}{\sqrt{2}}\left(\eta_{1}-i \eta_{2}\right)$ into Eq. (1) is adopted. As a result, electrons within $f$ and $d_{1}$ beyond the normal tunneling $t$ between them, become bounded as a Cooper pair with binding energy $\Delta$. It is worth mentioning that $\Delta$ used here refers to the coupling term as indicated in Fig 1(a) and it does not represent the superconducting gap of the $p$-wave Kitaev chains. However, the emergence of such a parameter can be understood as consequence of the proximity effect arising from the $s$-wave superconductors hosting the Kitaev chains. We would like to clarify that if the actual gauge imposed above were another, the QPT as well as the electric current switch feature would be triggered by fixing $t_{L}$ and increasing slightly, for instance, $t_{R}$ (or vice-versa). Such a tuning of the amplitudes $t_{L}$ and $t_{R}$ nowadays is completely possible, thus turning the feasibility of the proposal concrete experimentally.

\section{A. Green's functions}

To calculate the conductance $G$, which depends upon the retarded Green's functions $\tilde{\mathcal{G}}_{\mathcal{A B}}$ in the energy domain $\varepsilon$, with $\mathcal{A}$ and $\mathcal{B}$ as fermionic operators belonging to the Hamiltonian $\mathcal{H}$, we should employ the equation-ofmotion $\left(\right.$ EOM) method ${ }^{27}$ summarized as follows $\omega \tilde{\mathcal{G}}_{\mathcal{A B}}=$ $\left(\varepsilon+i 0^{+}\right) \tilde{\mathcal{G}}_{\mathcal{A B}}=\left[\mathcal{A}, \mathcal{B}^{\dagger}\right]_{+}+\tilde{\mathcal{G}}_{\left[\mathcal{A}, \mathcal{H}_{i}\right] \mathcal{B}}$. By applying the EOM on $\mathcal{G}_{d_{j} d_{l}}=-\frac{i}{\hbar} \theta(\tau) \operatorname{Tr}\left\{\varrho\left[d_{j}(\tau), d_{l}^{\dagger}(0)\right]_{+}\right\}$here expressed 
in terms of the density matrix $\varrho$ for Eq. (1) and the Heaviside function $\theta(\tau)$, we change to the energy domain $\varepsilon$ and obtain the following relation:

$$
\left(\varepsilon-\varepsilon_{j}-\Sigma-\delta_{j 1} \Sigma_{\mathrm{MQPs}}\right) \tilde{\mathcal{G}}_{d_{j} d_{l}}=\delta_{j l}+\Sigma \sum_{\tilde{l} \neq j} \tilde{\mathcal{G}}_{d_{i} d_{l}},
$$

with $\Sigma=-(\sqrt{x}+i)(1+x)^{-1} \Gamma, x=\left(\pi \rho_{0} V_{S D}\right)^{2}$, the MQPs self-energy $\Sigma_{\mathrm{MQPs}}=K(t, \Delta)+(2 t \Delta)^{2} K \tilde{K}$, where $K(t, \Delta)=\left[\varepsilon^{2}+2 i \varepsilon 0^{+}-\left(0^{+}\right)^{2}\right]^{-1} \omega\left(t^{2}+\Delta^{2}\right), K=\left[\varepsilon^{2}+\right.$ $\left.2 i \varepsilon 0^{+}-\left(0^{+}\right)^{2}\right]^{-1} \omega, \tilde{K}=\left[\varepsilon+\varepsilon_{1}+\bar{\Sigma}-K(t, \Delta)\right]^{-1} K$ and $\bar{\Sigma}_{\tilde{G}}$ as the complex conjugate of $\Sigma$. Thus the solution of $\tilde{\mathcal{G}}_{d_{j} d_{l}}$ provides

$$
\tilde{\mathcal{G}}_{d_{1} d_{1}}=\frac{1}{\varepsilon-\varepsilon_{1}-\Sigma-\Sigma_{\mathrm{MQPs}}-\mathcal{C}_{2}}
$$

as the Green's function of the QD 1, with $\mathcal{C}_{j}=\Sigma^{2}(\varepsilon-$ $\left.\varepsilon_{j}-\Sigma\right)^{-1}$ as the self-energy due to the presence of the $j^{\text {th }} \mathrm{QD}$. In the case of the QD 2, we have

$$
\tilde{\mathcal{G}}_{d_{2} d_{2}}=\frac{1-\tilde{\mathcal{G}}_{d_{1} d_{1}}^{0} \Sigma_{\mathrm{MQPs}}}{\varepsilon-\varepsilon_{2}-\Sigma-\frac{\tilde{\mathcal{G}}_{d_{1} d_{1}}^{0}}{\mathcal{\mathcal { G }}_{d_{2} d_{2}}} \Sigma_{\mathrm{MQPs}}-\mathcal{C}_{1}},
$$

where $\tilde{\mathcal{G}}_{d_{1} d_{1}}^{0}=\left(\varepsilon-\varepsilon_{1}-\Sigma\right)^{-1}$ and $\tilde{\mathcal{G}}_{d_{2} d_{2}}^{0}=\left(\varepsilon-\varepsilon_{2}-\right.$ $\Sigma)^{-1}$ represent the corresponding Green's functions for the single QD system without MQPs. The mixed Green's functions are $\tilde{\mathcal{G}}_{d_{2} d_{1}}=\Sigma\left(\varepsilon-\varepsilon_{2}-\Sigma\right)^{-1} \tilde{\mathcal{G}}_{d_{1} d_{1}}$ and $\tilde{\mathcal{G}}_{d_{1} d_{2}}=$ $\Sigma\left(\varepsilon-\varepsilon_{1}-\Sigma-\Sigma_{\mathrm{MQPs}}\right)^{-1} \tilde{\mathcal{G}}_{d_{2} d_{2}}$.

\section{B. Conductance}

In what follows we derive the LandauerBüttiker formula for the zero-bias conductance $G=\left(e^{2} / h\right) \mathcal{T}(\varepsilon=0, t, \Delta)$ at temperature $T=0^{27}$. Such a quantity is a function of the transmittance $\mathcal{T}(\varepsilon, t, \Delta)$ as follows:

$$
\begin{aligned}
\mathcal{T}(\varepsilon, t, \Delta) & =\mathcal{T}_{b}+2 \sqrt{\mathcal{T}_{b} \mathcal{R}_{b}} \tilde{\Gamma} \sum_{j \tilde{j}} \operatorname{Re}\left\{\tilde{\mathcal{G}}_{d_{j} d_{\tilde{j}}}(\varepsilon)\right\} \\
& -\left(1-2 \mathcal{T}_{b}\right) \tilde{\Gamma} \sum_{j \tilde{j}} \operatorname{Im}\left\{\tilde{\mathcal{G}}_{d_{j} d_{\tilde{j}}}(\varepsilon)\right\} \\
& =\mathcal{T}_{b}+\sum_{j} \mathcal{T}_{j j}(\varepsilon, t, \Delta)+\sum_{j} \mathcal{T}_{j \bar{j}}(\varepsilon, t, \Delta),
\end{aligned}
$$

where $j, \tilde{j}=1,2$ and $\bar{j}=1,2$ respectively for $j=2,1$ as labels to correlate distinct QDs, $\tilde{\Gamma}=\frac{\Gamma}{1+x}$ is an effective dot-lead coupling, $\mathcal{T}_{b}=\frac{4 x}{(1+x)^{2}}$ represents the background transmittance and $\mathcal{R}_{b}=1-\mathcal{T}_{b}=\frac{(1-x)^{2}}{(1+x)^{2}}$ is the corresponding reflectance, both in the absence of the QDs and MQPs, $\mathcal{T}_{j j}(\varepsilon, t, \Delta)$ gives the transmittance through the $j^{\text {th }} \mathrm{QD}$, while the crossed term $\mathcal{T}_{j \bar{j}}(\varepsilon, t, \Delta)$ accounts for interference processes between these dots. For $t=\Delta$, we recover the Green's functions derived in Refs. 28] and 29]. In such works, we point out that the profiles of $\mathcal{T}(\varepsilon, t=\Delta)$ as a function of the single particle energy $\varepsilon$ for a given symmetric detuning $\Delta \varepsilon$ of the QDs appear analyzed in great detail, since this case corresponds to the unique presence of a Kitaev chain. Thus, a robust Majorana ZBP is found when $\mathcal{T}_{b}=0$ and the corresponding dip for $\mathcal{T}_{b}=1$ is observed. We then suggest the reader to see Refs. [28] and [29] where $\mathcal{T}(\varepsilon, t=\Delta)$ versus $\varepsilon$ for finite values of $\Delta \varepsilon$ can be found. In the limit of $t \neq \Delta$, we have checked that the Majorana ZB anomaly (peak or dip) is not verified. For this latter case, see for instance Fig $2(\mathrm{c})$, wherein $\mathcal{T}_{11}(\varepsilon, t \neq \Delta)$ and $\mathcal{T}_{21}(\varepsilon, t \neq \Delta)+\mathcal{T}_{12}(\varepsilon, t \neq \Delta)$ as functions of $\varepsilon$ appear explicitly for several $\Delta \varepsilon$. Additionally, it is worth noticing that the QPT as well as the electric current switch feature of the transmittance, just emerge when $\varepsilon=0$ and as a function of $\Delta \varepsilon$.

\section{RESULTS AND DISCUSSION}

Based on the Green's functions derived previously together with Eq.(5) for the total transmittance $\mathcal{T}(\varepsilon, t, \Delta)$, we focus on two cases ruled by analytical expressions here determined for $\mathcal{T}(\varepsilon=0, t \neq \Delta, \Delta \varepsilon)$ and $\mathcal{T}(\varepsilon=0, t=\Delta, \Delta \varepsilon)$, as follows: the "Switch" regime for the current, sketched in details in Figs2(a) and 3(a), which rises when we set the device to $\mathcal{T}_{b}=0, \varepsilon=0$ (zero-bias) with $\varepsilon_{1}=\frac{\Delta \varepsilon}{2}$ and $\varepsilon_{2}=-\frac{\Delta \varepsilon}{2}$ as the symmetric detuning for the QDs; Off the "Switch" regime, where just $\mathcal{T}_{b}=1$ is changed.

It is worth mentioning that the control parameter of the QPT is given by the ratio $t / \Delta$. Thus from now on, in Figs 2,3 and 4 we identify by the label "numerical" those curves determined by Eq. (51) by considering $t=6 \Gamma$ with $\Delta=7 \Gamma$ and $t=\Delta=6 \Gamma$ respectively for the limits $t \neq \Delta$ and $t=\Delta$, otherwise the analytical expressions for $\mathcal{T}(\varepsilon=0, t \neq \Delta, \Delta \varepsilon)$ and $\mathcal{T}(\varepsilon=0, t=\Delta, \Delta \varepsilon)$ here obtained appear with the label "analytical", which are functions indeed explicitly independent on $t$ and $\Delta$. Here the energy scale adopted in the simulations is the Anderson parameter $\Gamma^{24}$.

\section{A. Sharpness of the switching action}

We highlight that the analytical expressions that we have found, which will appear later on, in the limits $t \neq \Delta$ and $t=\Delta$, wondrously reveals a universal behavior by means of the independence on the parameters $t$ and $\Delta$. Consequently, it means that numerically speaking, i.e., without performing the aforementioned analytical simplifications in Eq.(15) for $\mathcal{T}(\varepsilon, t, \Delta)$, the emulation of a weak suppression of the coupling between the QD 1 and Kitaev chain 2 , can be realized by a slight change in $\Delta$ by fixing $t$ within such an equation, which then makes the 
system to undergo a QPT, since the phases $t \neq \Delta$ and $t=\Delta$ are not smoothly connected being characterized by an absence of a crossover.

As a result, there is a sudden change in the transmittance profile as we will see, in which each phase is recognized by the derived analytical expression describing $\mathcal{T}(\varepsilon=0, t \neq \Delta, \Delta \varepsilon)$ and $\mathcal{T}(\varepsilon=0, t=\Delta, \Delta \varepsilon)$, respectively. It means that, the sharpness of the switching action of $\mathcal{T}(\varepsilon=0, t, \Delta, \Delta \varepsilon)$ is given by a Dirac delta behavior pinned at $t=\Delta$ when $t-\Delta$ is varied.

\section{B. The "Switch" regime}

By considering $\mathcal{T}_{b}=0$ and $t \neq \Delta$, we mimic both the Kitaev chains of Fig 2 coupled to the QD 1, then allowing the current to switch through the lower QD only, where we see diagrammatically by the orange arrows, the current crossing solely the QD 2 of panel (a) of the same figure. Within this regime, the total transmittance reduces to

$$
\mathcal{T}(\varepsilon=0, t \neq \Delta, \Delta \varepsilon)=\mathcal{T}_{22}=\frac{\Gamma^{2}}{\Gamma^{2}+\frac{1}{4} \Delta \varepsilon^{2}}
$$

as outlined in Fig 2(b), where we recognize $\mathcal{T}_{22}=1$ for the QDs on resonance $(\Delta \varepsilon=0)$ that connect the metallic leads via the Fermi energy $(\varepsilon=0)$ through the QD 2 and $\mathcal{T}_{22}=0$, the off-resonance case $(\Delta \varepsilon \gg \Gamma$ and $\Delta \varepsilon \ll-\Gamma)$ wherein such a connection is found truncated.

The unique contribution from $\mathcal{T}_{22}$ to the total transmittance of the system, then lies on the features $\mathcal{T}_{11}(\varepsilon=0, t \neq \Delta, \Delta \varepsilon)=0$ and $\mathcal{T}_{12}(\varepsilon=0, t \neq \Delta, \Delta \varepsilon)+$ $\mathcal{T}_{21}(\varepsilon=0, t \neq \Delta, \Delta \varepsilon)=0$ that do ensure a null contribu-

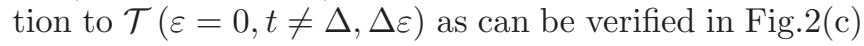
as a function of $\varepsilon$, for different $\Delta \varepsilon$ values.

The partial transmittance $\mathcal{T}_{11}(\varepsilon=0, t \neq \Delta, \Delta \varepsilon)=0$ reflects that the QD 1 perceives both the two MQPs at the edges of the Kitaev chains as an ordinary fermionic zero mode, in such a way that the splitting of this mode occurs in $\mathcal{T}_{11}$, which opens a superconducting gap as pointed out in Fig2(c), which prevents the current as a result. On the other hand, $\mathcal{T}_{12}(\varepsilon=0, t \neq \Delta, \Delta \varepsilon)+$ $\mathcal{T}_{21}(\varepsilon=0, t \neq \Delta, \Delta \varepsilon)=0$ encode the scattering of electrons traveling forth and back between the upper and lower QDs, which are phase shifted by $\pi$, i.e., $\mathcal{T}_{12}(\varepsilon=0, t \neq \Delta, \Delta \varepsilon)=-\mathcal{T}_{21}(\varepsilon=0, t \neq \Delta, \Delta \varepsilon)$ canceling the net current through this path.

The case $t=\Delta$ switches the upper QD suddenly, thus characterizing the QPT found in panel (a) of Fig 3 , then yielding solely

$$
\mathcal{T}(\varepsilon=0, t=\Delta, \Delta \varepsilon)=\mathcal{T}_{11}=\frac{1}{2}+\frac{1}{2} \frac{\Gamma^{2}}{\Gamma^{2}+\Delta \varepsilon^{2}}
$$

as it appears in Fig 3(b), which differently from Fig[2(b), gives $\mathcal{T}_{11}=0.5$ in the limits $\Delta \varepsilon \gg \Gamma$ and $\Delta \varepsilon \ll-\Gamma$ : see the half-spheres outlined in the panel (b) of Fig 3
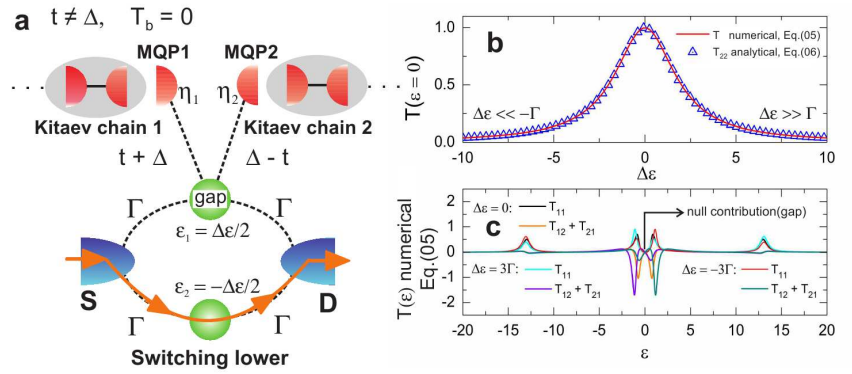

Figure 2. (Color online) The "Switch" regime $\mathcal{T}_{b}=0$ : (a) The QD 1 perceives the two MQPs $\eta_{1}$ and $\eta_{2}$ (an ordinary fermion), in particular with $t \neq \Delta$. (b) As a result of (a), just the zero-bias transmittance $(\varepsilon=0) \mathcal{T}_{22}$ contributes to the total system transmittance $\mathcal{T}$ as a function of the symmetric detuning $\Delta \varepsilon$ for the QDs. (c) The transmittances $\mathcal{T}_{11}$ and $\mathcal{T}_{12}+\mathcal{T}_{21}$ exhibit a superconducting gap to prevent the current.
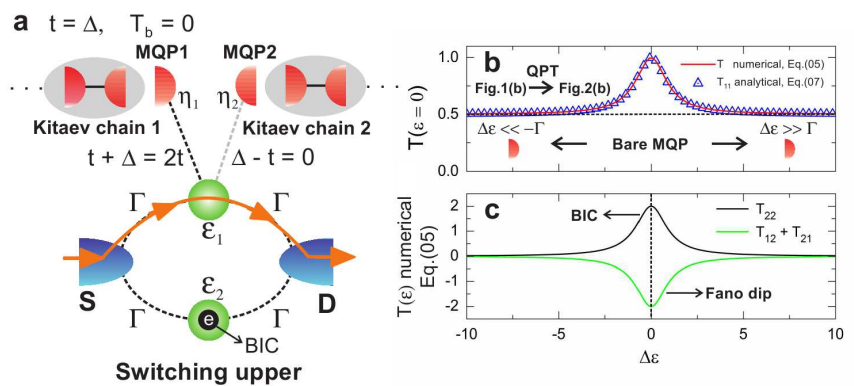

Figure 3. (Color online) The "Switch" regime $\mathcal{T}_{b}=0$ : (a) The current is switched upper (orange arrows) through the QD 1, when such a dot perceives solely the MQP $\eta_{1}$ for $t=\Delta$. (b) A QPT occurs due to the system abrupt change from $t \neq \Delta$ to $t=\Delta$, giving rise to a transmittance profile fully distinct with respect to that found in Fig 2(b). (c) In this case, the transmittance $\mathcal{T}_{22}$ is canceled by a Fano dip in $\mathcal{T}_{12}+\mathcal{T}_{21}$. An electron $e$ is trapped within the lower QD as a BIC.

for such a pictorial representation, which points out the zero mode of the MQP at the edge in the Kitaev chain 1 that leaked into the QD $1 \frac{17}{17}$. In such a case, the path via $\mathcal{T}_{11}$ is chosen due to the emerging Fano dip 30,31 in $\mathcal{T}_{12}(\varepsilon=0, t=\Delta, \Delta \varepsilon)+\mathcal{T}_{21}(\varepsilon=0, t=\Delta, \Delta \varepsilon)$ that results in a perfect destructive interference with the resonance lineshape of $\mathcal{T}_{22}(\varepsilon=0, t=\Delta, \Delta \varepsilon)$ as it appears in Fig 3(c), which cancels the possibility of other paths for the current through the system. This perfect cancelation points out that an electron is trapped within the lower QD as a BIC, which then blocks the current to cross from the source towards drain. We highlight that the Fano interference mechanism of the BIC emergence can be found discussed in great detail in Refs. 32] and [33] for the graphene system. Particularly, the BIC phenomenon here verified occurs when the nature of the device is purely due to a MQP.

Thus, distinctly from the situation $t \neq \Delta$ for an ordinary fermion present, where the absence of states in QD 1 due to a gap that prevents the current flow, in the 


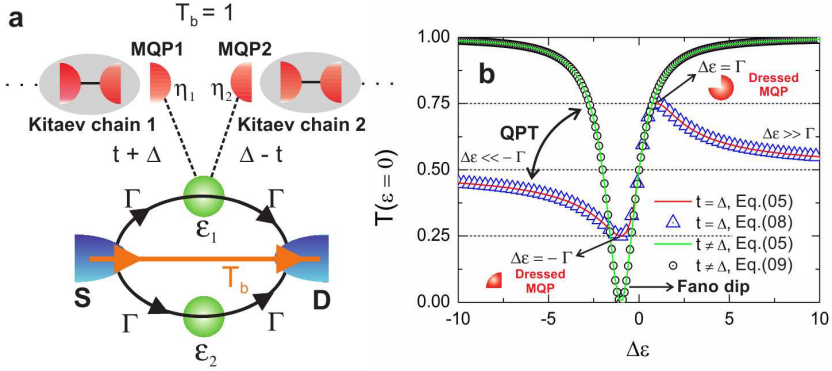

Figure 4. (Color online) Off the "Switch" regime $\mathcal{T}_{b}=1$ : (a) The lead-lead coupling $\mathcal{T}_{b}$ now exists and the current crosses the QDs as well as the middle region between the leads. (b) Even without the current switch feature in the device, the QPT remains made explicit by the abrupt change in the transmittance profile, wherein novel MQP signatures rise given by $\mathcal{T}=0.25$ and $\mathcal{T}=0.75$, respectively for $\Delta \varepsilon=-\Gamma$ and $\Delta \varepsilon=\Gamma$.

$t=\Delta$ regime a single electron is bounded to the QD 2, which does not allow extra electrons to pass as aftermath of the electronic state of this QD, which is filled indefinitely due to the BIC phenomenon that puts into such a dot an electron with an infinite lifetime.

\section{Off the "Switch" regime}

For this regime we have $\mathcal{T}_{b}=1$ and the absence of the current switch feature as depicted in Fig प(a), where the current crosses simultaneously the QDs and the middle region between the metallic leads, once we have checked that all terms of Eq.(5) are relevant to the total transmittance. Here Fig $4(\mathrm{~b})$ exhibits the QPT wherein an abrupt change in the transmittance profile still remains, i.e.,

$$
\mathcal{T}(\varepsilon=0, t=\Delta, \Delta \varepsilon)=\frac{1}{2}+\frac{1}{2} \frac{\Delta \varepsilon \Gamma}{\Gamma^{2}+\Delta \varepsilon^{2}}
$$

and

$$
\mathcal{T}(\varepsilon=0, t \neq \Delta, \Delta \varepsilon)=1-\frac{\Gamma^{2}}{(\Gamma+\Delta \varepsilon)^{2}+\Delta \varepsilon^{2}},
$$

due to the change from the case $t=\Delta$ to $t \neq \Delta$. The latter function describes the situation in which the QD 1 perceives the Kitaev chains 1 and 2 as an ordinary fermion, being characterized by $\mathcal{T}=1$ when the dots are found very far from the resonance $(\Delta \varepsilon \gg \Gamma$ and $\Delta \varepsilon \ll$ $-\Gamma$ ) recovering the background transmittance $\mathcal{T}_{b}=1$ of the leads, in addition to the point $\Delta \varepsilon=-\Gamma$ where $\mathcal{T}=0$ giving rise to a perfect Fano destructive interference.

By entering into $t=\Delta$ regime, the transmittance alters drastically its profile thus making explicitly the QPT as a result of the isolated MQP 1 , as can be verified in the panel (b) of the same figure.

It is worth mentioning that, in particular, we predict well-established fractional values for the transmittance. From the expression $\mathcal{T}(\varepsilon=0, t=\Delta, \Delta \varepsilon)$ above, one can show that especially for the detuning of the QDs $\Delta \varepsilon= \pm \Gamma$, we find $\mathcal{T}(\varepsilon=0, t=\Delta, \Delta \varepsilon=-\Gamma)=0.25$ and $\mathcal{T}(\varepsilon=0, t=\Delta, \Delta \varepsilon=\Gamma)=0.75$, which point out "dressed" MQPs traveling through the interferometer, appearing depicted by the incomplete spheres in Fig 4(b) in order to indicate pictorially the emergence of such quasiparticles. These novel fractional values for the transmittance exactly placed at $\Delta \varepsilon=-\Gamma$ and $\Delta \varepsilon=\Gamma$ are then helpful to recognize that one isolated MQP lies on the Kitaev chain 1.

\section{CONCLUSIONS}

In summary, by assuming $\mathcal{T}_{b}=0$ we have the phase $t \neq \Delta$ with $\mathcal{T}=\mathcal{T}_{22}$ (switching lower) when the QD 1 "feels" both the MQPs at the edges of the Kitaev chains as an ordinary fermion, followed by the phase $t=\Delta$ wherein $\mathcal{T}=\mathcal{T}_{11}$ (switching upper) for this dot solely connected to the Kitaev chain 1, when the system has a single MQP isolated. Hence, the sudden change in the path for the current, which does not have nothing to do with the elusive ZBP signature, is then triggered by the QPT here addressed and serves not only to reveal a MQP isolated in the system, but also to propose as an application a current switch assisted by MQPs. Furthermore for $\mathcal{T}_{b}=1$, we have found the novel fractional values $G=0.25 e^{2} / h$ and $G=0.75 e^{2} / h$ in the conductance when the system is off the "Switch" regime, which can help to recognize a MQP.

\section{ACKNOWLEDGMENTS}

This work was supported by CNPq, CAPES, 2014/14143-0, 2015/23539-8, 2015/26655-9 São Paulo Research Foundation (FAPESP).
1 J. Alicea, Rep. Prog. Phys. 75, 076501 (2012).

2 S.R. Elliott and M. Franz, Rev. Mod. Phys. 87, 137 (2015).

${ }^{3}$ M. Leijnse and K. Flensberg, Semicond. Sci. Technol. 27 124003 (2012).

4 V. Mourik, K. Zuo, S.M. Frolov, S.R. Plissard, E.P.A.M. Bakkers, and L.P. Kouwenhoven, Science 336, 1003 (2012).
5 S.N.-Perge, I.K. Drozdov, J. Li, H. Chen, S. Jeon, J. Seo, A.H. MacDonald, B.A. Bernevig, and A. Yazdani, Science 346, 602 (2014).

6 J. Liu, A.C. Potter, K.T. Law, and P.A. Lee, Phys. Rev. Lett. 109, 267002 (2012). 
7 E. Dumitrescu, B. Roberts, S. Tewari, J.D. Sau, and S.Das Sarma, Phys. Rev. B 91, 094505 (2015).

8 P.A. Lee, Science 346, 545 (2014).

9 A.Y. Kitaev, Phys. Usp. 44, 131 (2001).

10 F. Iemini, L. Mazza, D. Rossini, R. Fazio, and S. Diehl, Phys. Rev. Lett. 115, 156402 (2015).

11 D. Roy, C. J. Bolech, and N. Shah, Phys. Rev. B 86, 094503 (2012).

12 A.A. Zyuzin, D. Rainis, J. Klinovaja, and D. Loss, Phys. Rev. Lett. 111, 056802 (2013).

13 R. Pawlak, M. Kisiel, J. Klinovaja, T. Meier, S. Kawai, T. Glatzel, D. Loss, and E. Meyer, arXiv:1505.06078v2 (2015).

14 S.M. Albrecht, A.P. Higginbotham, M. Madsen, F. Kuemmeth, T.S. Jespersen, J. Nygard, P. Krogstrup, C.M. Marcus, Nature 531, 206 (2016).

15 A. Zazunov, P. Sodano, and R. Egger, New J. Phys 15, 035033 (2013).

16 D.E. Liu and H.U. Baranger, Phys. Rev. B 84, 201308(R) (2011).

17 E. Vernek, P.H. Penteado, A.C. Seridonio, and J.C. Egues, Phys. Rev. B 89, 165314 (2014).

18 L.S. Ricco, Y. Marques, F.A. Dessotti, R.S. Machado, M. de Souza, and A.C. Seridonio, Phys. Rev. B 93, 165116 (2016).

19 J. von Neumann and E. Wigner, Phys. Z. 30, 465 (1929).

${ }^{20}$ F.H. Stillinger and D.R. Herrick, Phys. Rev. A 11, 446 (1975).

21 F. Capasso, C. Sirtori, J. Faist, D.L. Sivco, S.-N.G. Chu, and A.Y. Cho, Nature 358, 565 (1992).
22 A.R. Akhmerov, J.P. Dahlhaus, F. Hassler, M. Wimmer, and C.W.J. Beenakker, Phys. Rev. Lett. 106, 057001 (2011).

23 D. Rainis and D. Loss Phys. Rev. B 85, 174533 (2012).

24 P.W. Anderson, Phys. Rev. 124, 41 (1961).

25 Y. Cao, P. Wang, G. Xiong, M. Gong, and X.-Q. Li, Phys. Rev. B 86, 115311 (2012).

26 W.-J. Gong, S.-F. Zhang, Z.-C. Li, G. Yi, and Y.S. Zheng, Phys. Rev. B 89, 245413 (2014).

27 H. Haug and A.P. Jauho, Quantum Kinetics in Transport and Optics of Semiconductors, Springer Series in SolidState Sciences Vol. 123 (Springer, New York, 1996).

28 A.C. Seridonio, E.C. Siqueira, F.A. Dessotti, R.S. Machado, and M. Yoshida, J. Appl. Phys. 115, 063706 (2014).

29 F.A. Dessotti, L.S. Ricco, M. de Souza, F.M. Souza, and A.C. Seridonio, J. Appl. Phys. 116, 173701 (2014).

${ }^{30}$ U. Fano, Phys. Rev. 124, 1866 (1961).

31 A.E. Miroshnichenko, S. Flach, and Y.S. Kivshar, Rev. Mod. Phys. 82, 2257 (2010).

32 L.H. Guessi, R.S. Machado, Y. Marques, L.S. Ricco, K. Kristinsson, M. Yoshida, I.A. Shelykh, M. de Souza, and A.C. Seridonio, Phys. Rev. B 92, 045409 (2015).

33 L.H. Guessi, Y. Marques, R.S. Machado, L.S. Ricco, K. Kristinsson, M.S. Figueira, I.A. Shelykh, M. de Souza, and A.C. Seridonio, Phys. Rev. B 92, 245107 (2015). 